\title{
Report on the New NBS Determination of the Proton Gyromagnetic Ratio
}

\author{
EDWIN R. WILLIAMS, GEORGE R. JONES, MEMBER, IEEE, JUN-SHOU SONG, WILLIAM D. PHILLIPS, \\ AND PAUL T. OLSEN, MEMBER, IEEE
}

\begin{abstract}
We describe a new measurement of the proton gyromagnetic ratio in $\mathrm{H}_{2} \mathrm{O}, \gamma_{p}^{\prime}$, now in progress at NBS, including the construction of a single layer precision solenoid, the measurement of its dimensions by an inductive technique, and our latest dimensional measurement results. We also discuss other improvements made since our last $\gamma_{p}^{\prime}$ determination.
\end{abstract}

\section{INTRODUCTION}

$\mathrm{T}$ HE measurement of the proton gyromagnetic ratio in $\mathrm{H}_{2} \mathrm{O}$ by the weak field method involves measuring two quantities. First, one measures the dimensions of a precision solenoid and calculates the magnetic field produced when a known current is passed through the solenoid windings. Second, one measures the precession frequency of protons in a sample of $\mathrm{H}_{2} \mathrm{O}$ placed in this calculable field using standard nuclear magnetic resonance (NMR) techniques. The ratio of the NMR angular frequency to the calculated flux density is $\gamma_{p}^{\prime}($ low). The dimensional measurements have always been the more difficult part of the determination.

In this paper we describe our present experiment which employs a newly constructed precision solenoid. We also discuss the major changes we have made in our previous experiment [1] and why we expect these changes to improve our accuracy from $0.2 \mathrm{ppm}$ to better than $0.05 \mathrm{ppm}$.

The major motivation for a new $\gamma_{p}^{\prime}($ low $)$ experiment is to help test basic physical theories via the fundamental constants. In particular, the fine structure constant, $\alpha$, can be obtained from the Josephson effect measurement of $2 e / h$ and $\gamma_{p}^{\prime}($ low $)$ via the following equation:

$$
\alpha^{-2}=\frac{c}{4 R_{\infty}} \frac{1}{\left(\Omega_{\mathrm{NBS}} / \Omega\right)} \frac{\mu_{p}^{\prime}}{\mu_{B}} \frac{(2 e / h)_{\mathrm{NBS}}}{\gamma_{p}^{\prime}(\text { low })_{\mathrm{NBS}}} .
$$

Here $c$ is the speed of light in vacuum; $R_{\infty}$ is the Rydberg constant for infinite mass; $\Omega_{\mathrm{NBS}} / \Omega$ is the ratio of the National Bureau of Standards (NBS) as-maintained ohm to the absolute or SI (International System) ohm; $\mu_{p}^{\prime} / \mu_{B}$ is the magnetic moment of the proton in units of the Bohr magneton (through-

Manuscript received August 20, 1984. This work was supported in part by the U.S. Department of Energy and the U.S. Air Force Office of Scientific Research.

E. R. Williams, G. R. Jones, W. D. Phillips, and P. T. Olsen are with the National Bureau of Standards, Washington, DC.

J. S. Song is with the National Bureau of Standards, Washington, DC, as a Guest Scientist from Chongqing University, People's Republic of China. out, the prime indicates protons in a spherical sample of pure $\mathrm{H}_{2} \mathrm{O}$ at $\left.25^{\circ} \mathrm{C}\right) ;(2 e / h)_{\mathrm{NBS}}$ is the ratio of twice the elementary charge to the Planck constant measured in terms of the NBS as-maintained volt using the ac Josephson effect; and $\gamma_{p}^{\prime}(\text { low })_{\mathrm{NBS}}$ is the proton gyromagnetic ratio measured in terms of the NBS as-maintained ampere.

The accurate determination of $\gamma_{p}^{\prime}$ (low) has become even more important with the discovery of the quantum Hall effect. The pertinent equation to obtain $\alpha$ from the quantized Hall resistance $R_{H}$ is

$$
\alpha^{-1}=\frac{2}{\mu_{0} c} \frac{\Omega_{\mathrm{NBS}}}{\Omega}\left(R_{H}\right)_{\mathrm{NBS}}
$$

where $\mu_{0}$ is the permeability of free space. Equations (1) and (2) can be combined to eliminate the quantity $\Omega_{\mathrm{NBS}} / \Omega$, yielding

$$
\alpha^{-3}=\frac{1}{2 \mu_{0} R_{\infty}} \frac{\mu_{p}^{\prime}}{\mu_{B}} \frac{(2 e / h)_{\mathrm{NBS}}}{\gamma_{p}^{\prime}(\mathrm{low})_{\mathrm{NBS}}}\left(R_{H}\right)_{\mathrm{NBS}}
$$

Equation (3) is obviously not independent of (2) but represents an alternative path to obtain $\alpha$ from the Hall resistance. However, the cube root dependence of (3) helps reduce the uncertainty in $\alpha$. Of course, all three results could be combined together to obtain a least squares value for $\alpha$. It is clear that by measuring $\gamma_{p}^{\prime}$ and $\Omega_{\mathrm{NBS}} / \Omega$ more accurately, we can improve our understanding of the ac Josephson effect and the quantum Hall effect, while testing quantum electrodynamics which requires $\alpha$ for the evaluation of its theoretical expressions (e.g., for the anomalous moment of the electron). Since the completion of our last $\gamma_{p}^{\prime}$ experiment three other experimental results have been published. (These experiments are reviewed in [2]). The National Physical Laboratory in England and the Mendelyeev Institute of Metrology in the USSR have reported values of $\gamma_{p}^{\prime}$ that are in complete disagreement with our 1979 result (10-15 standard deviation discrepancies). In contrast, the Chinese value obtained at the National Institute of Metrology agrees with our value. A new determination of $\gamma_{p}^{\prime}$ should shed light on these discrepancies.

The dominant source of uncertainty in our previous experiment was the measurement of the diameter of the solenoid. We attempted to eliminate the need for a diameter measurement by using a special distribution of currents [1], but this technique does not work well on short solenoids. We have, therefore, constructed a long solenoid that will allow us to eliminate the need to measure the diameter. 


\section{Construction of a Long SINGLE-LAYER SOLENOID}

We fabricated the new 2.2-m long 0.3-m diam, fused silica solenoid by lapping techniques developed specifically for precision solenoid construction at NBS by Curtis, Moon, and Sparks in 1932 [3]. In about 1958, Driscoll perfected the technique while lapping the solenoid used in our previous determination. Because of the critical role of the solenoid in determining $\gamma_{p}^{\prime}$ (low), we feel it is of interest to summarize briefly its fabrication.

We started with a rough cylindrical form of fused silica (actually magnetically purified silica sand fused together) $2.2 \mathrm{~m}$ long, with a $25-\mathrm{mm}$ wall thickness, manufactured by U.S. Fused Quartz Company. ${ }^{1}$ The form was first ground into a cylinder using a large lathe on which we mounted a grinding wheel. A special lathe was then constructed for the lapping process by cutting an old lathe in the middle and extending it to handle the 2.2-m cylinder. This was necessary because we could not use the large lathe for the time consuming lapping process. We lapped the cylinder with a lap that made contact on two generators of the cylinder $\pm 45^{\circ}$ from the top. This lap was constrained so that it would not rotate with the cylinder but was free to sit on the top of the cylinder. With grit applied, the lap was gently guided up and down the cylinder. Frequently the cylinder had to be cleaned and measurements taken of the diameter along its length. The time spent lapping various areas was adjusted so as to obtain a diameter constant to a few micrometers. A slight ellipticity $(\sim 3 \mu \mathrm{m})$ was detected and corrected by painting selected areas where less lapping was desired.

This cylinder was then mounted in the large lathe, and a helix of 24 threads/inch was ground 0.3-mm deep. It was then returned to the lapping lathe where a special lap was used to smooth the helical thread. To make this lap we machined an inside thread into a $1-\mathrm{cm}$ thick brass ring such that this ring could, in principle, be threaded onto the $0.3-\mathrm{m}$ diam helix ground in the cylinder. The brass ring, which was $10-\mathrm{cm}$ long, was then cut along the axial direction into short pieces $3-\mathrm{cm}$ wide. A total of 46 of the $3 \mathrm{~cm} \times 10 \mathrm{~cm}$ lap segments was spaced along four generators of the cylinder. Two generators were located at $\pm 60^{\circ}$ from the vertical, and two were at $\pm 30^{\circ}$. The segments were then epoxied to a lap form made of fused silica, while the lap segments were held with their threads seated securely in the grooves of the cylinder while spaced along the four generators.

The final lapping then proceeded as grit was applied to the rotating cylinder, and this 46 segment lap was driven back and forth along the top of the cylinder by the helical groove. The lapping process, of course, averages out the variation in the pitch of the helical groove. However, the diameter must be measured repeatedly and the lapping time adjusted at various positions along the cylinder to keep the diameter constant. A short lap made of 14 segments was used for correcting small variations in the diameter. The final helical groove took on a

\footnotetext{
${ }^{1}$ Mention of a manufacturer does not signify an endorsement by the U.S. National Bureau of Standards, nor does it imply that the item is the only or best item for any purpose.
}

smooth, nearly sinusoidal shape, and the lapping was stopped when a $0.8-\mathrm{mm}$ wire would fit tightly in this sinusoidal groove.

We then wound the solenoid by drawing wire through dies directly onto the cylindrical form. We started with $1-\mathrm{mm}$ diam oxygen-free pure copper that was plated with $1 \mu \mathrm{m}$ of gold. The final wire diameter of $0.8 \mathrm{~mm}$ was obtained after drawing through two dies.

The newly constructed solenoid was then taken to the nonmagnetic facility where the measurement of its dimensions and NMR frequency will be completed without any further movement of the solenoid. A shower of an insulating liquid, Fluorinert FC-43 manufactured by the 3M Corporation, is used to cool the solenoid and control its temperature to $0.02{ }^{\circ} \mathrm{C}$. The temperature coefficient of expansion of the fused silica is about $0.5 \mathrm{ppm} /{ }^{\circ} \mathrm{C}$.

\section{Measuring the Solenoid}

The solenoid has 2100 turns spaced about $1 \mathrm{~mm} / \mathrm{turn}$, and it is necessary to measure their position and changes in diameter to about $0.02 \mu \mathrm{m}$. We have constructed a new measuring apparatus for this purpose, but the principles upon which it is based are the same as in our earlier effort [4]. The following is a brief summation of the method.

The basic idea is to use a magnetic inductive technique to locate the current in the windings. Coils $\mathrm{A}$ and $\mathrm{A}^{\prime}$ in Fig. 1 form a linear differential transformer which locates the axial position of the current injected into selected (or activated) turns of the solenoid. Coils $\mathrm{A}$ and $\mathrm{A}^{\prime}$ are connected so that their output voltages cancel when centered on these activated turns, and a servo system locks the coil assembly to the null point with a precision of $0.02 \mu \mathrm{m}$. A mirror (corner cube $\mathrm{M}^{\prime}$ ) located in the center of the coil assembly is part of a laser interferometer system, with the reference mirror (M) located in the center of a second linear differential transformer.

We choose to activate 10 turns at one time, and under computer control move the current injector to any selected 10 turns until information about all 2100 turns of the solenoid is obtained. Coils $\mathrm{B}, \mathrm{B}^{\prime}$, and $\mathrm{C}$ form a radius-to-voltage transducer which measures the variations in the radius of the injected current loop. The voltage induced in coil $\mathrm{C}$ is inversely proportional to the radius of the activated turns. To detect small changes, we first "buck out" most of the voltage in coil $\mathrm{C}$ by using the two coils $\mathrm{B}$ and $\mathrm{B}^{\prime}$. At the same time that we are bucking out the voltage in coil $\mathrm{C}$, we are also increasing the sensitivity of this three-coil system to changes in the radius $R$ of the solenoid because the voltage in coils $B$ and $B^{\prime}$ increases when the radius $\mathrm{R}$ increases. This three-coil radius-to-voltage transducer is then calibrated by having a few turns of wire on both ends of the solenoid that are $50 \mu \mathrm{m}$ larger at one end and $60 \mu \mathrm{m}$ smaller at the other end.

In order to insure the valid measurement of the axial and radial variations, we use 10 other coils on the probe to measure the motion of the probe in the other degrees of freedom. Fig. 2 is a cross section of the probe in the plane of the in jected current. The coils labeled $V$ are wired in opposition, as are those labeled $\mathrm{H}$; they are used to measure the vertical and horizontal displacements using the gradient of the magnetic field produced by the activated turns. The coils VA are wired 


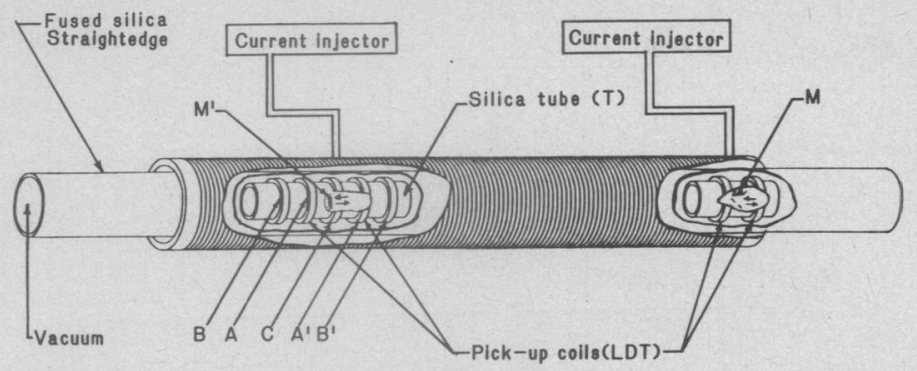

Fig. 1. Solenoid dimensional measurement system used to determine the axial position and radial variations of the wires. The five coils $\mathrm{A}, \mathrm{A}^{\prime}, \mathrm{B}, \mathrm{B}^{\prime}$, and $\mathrm{C}$ are attached to a silica tube, $\mathrm{T}$, and can be pushed or pulled along the axis of the solenoid. Coils $\mathbf{A}$ and $\mathrm{A}^{\prime}$ locate the axial position of the injected current and coils $\mathrm{B}, \mathrm{B}^{\prime}$, and $C$ form a diameter-to-voltage transducer. Mirrors $\mathbf{M}$ and $\mathbf{M}^{\prime}$ are part of a laser interferometer. The fused silica straight edge is used to guide the fivecoil probe and forms part of the vacuum chamber.

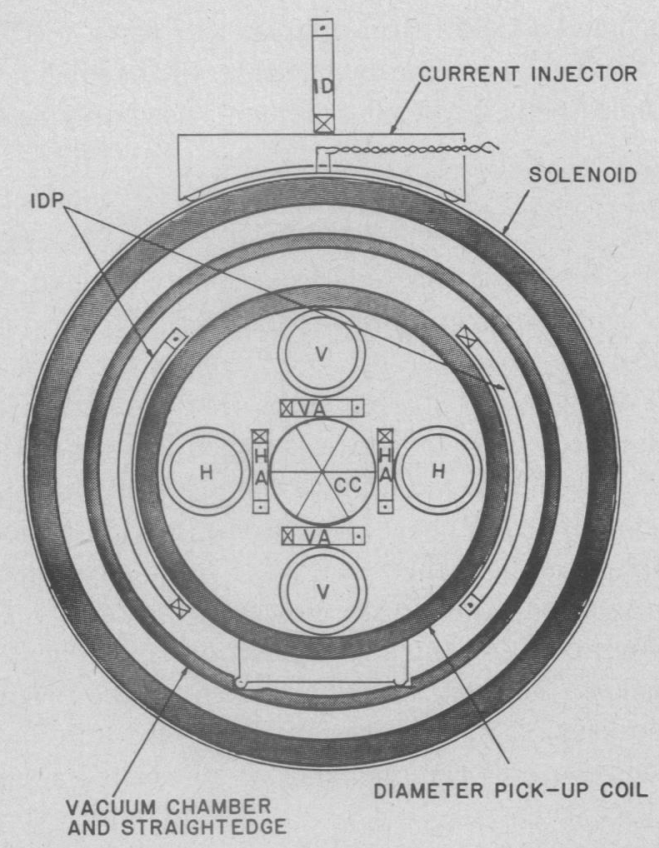

Fig. 2. Cross section of the pick-up probe and solenoid in the plane of the activated loop on the solenoid. A corner cube, CC, is located at the center. The coils marked V measure the vertical displacement, $\mathrm{H}$ the horizontal displacement, HA the horizontal angle, and VA the vertical angle. To measure the injector displacement, current is injected into the coil ID instead of the solenoid and the voltage in coil IDP is detected.

as one coil at the center and oriented orthogonally to the field of the activated turns. This pair measures the vertical angle (pitch), while HA measures the horizontal angle (yaw). The coil labeled ID is used in combination with the coil IDP to detect the current injector displacement with respect to the probe. Using these 10 coils, corrections are applied to the axial position and radial variation raw readings, in order to account for the fact that the straight edge that controls the position of the probe is not perfectly straight and that the current injector does not traverse the top of the solenoid perfectly. The radial variations voltage signal depends quadratically on the V, H, VA, and HA signals, and it is imperative that the probe is centered where this dependence is an extremum in order to reduce the effect of straight-edge imperfections.

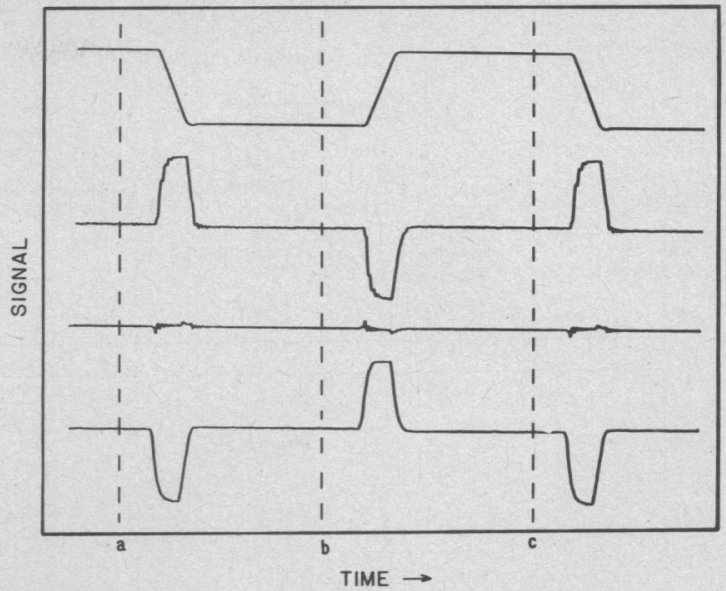

Fig. 3. Injection current and resulting signal. Upper trace: current injected into a portion of the solenoid. Second trace: voltage observed across the pick-up coils when the probe is displaced $+250 \mu \mathrm{m}$ from the center of the activated loop. Third trace: probe is centered. Bottom trace: probe is displaced $-250 \mu \mathrm{m}$. Letters indicate various times along the oscilloscope trace.

Another source of systematic error is very important and its elimination of interest. If the injected current is a sinewave, then an "end effect" error is introduced. Because this current induces a voltage in the other windings of the solenoid and because there is some capacitance between turns, some current flows in these windings, thereby producing an error signal in the pick-up coils [5]. This effect varies inversely with the square of the frequency and increases as the injection position approaches the end of the solenoid. We eliminate it by employing the special waveform for the injected current shown in the upper trace of Fig. 3. (This waveform is now generated by a digital waveform generator.) The output of the detector coil is just the derivative of the injected current. The second trace of Fig. 3 shows this output and results when the probe is $+250 \mu \mathrm{m}$ off null; the last trace is the output for $-250 \mu \mathrm{m}$ off null. In the next to last trace we are locked to the null. Using a lock-in amplifier with a flat frequency response, we can look at the integral of the output voltage from time a to time b, minus the integral from $b$ to $c$. This integral is proportional to the mutual inductance or total flux change in the pick-up coil. The transient signals, however, integrate to zero. The effectiveness of this method is demonstrated by either showing that the null position is independent of frequency or that it is independent of the phase of the lock-in. We find an error of less than $0.04 \mu \mathrm{m}$ for a phase shift of up to $90^{\circ}$.

The entire system is automated with a PDP-11 computer and CAMAC interface. The laser interferometer is a two-frequency, Zeeman-split laser system manufactured by Hewlett-Packard, and its frequency is measured against an iodine stabilized laser [6]. The entire interferometer is in a vacuum, so there is no correction due to the index of refraction of air. The laser and probe displacement are aligned by using split photo diodes. With these diodes we can keep the alignment error to less than $0.01 \mathrm{ppm}$.

Fig. 4 shows measurements of the new solenoid in the critical central region $( \pm 600 \mathrm{~mm})$. We are now concentrating on this region because the uncertainty in the calculated field due 


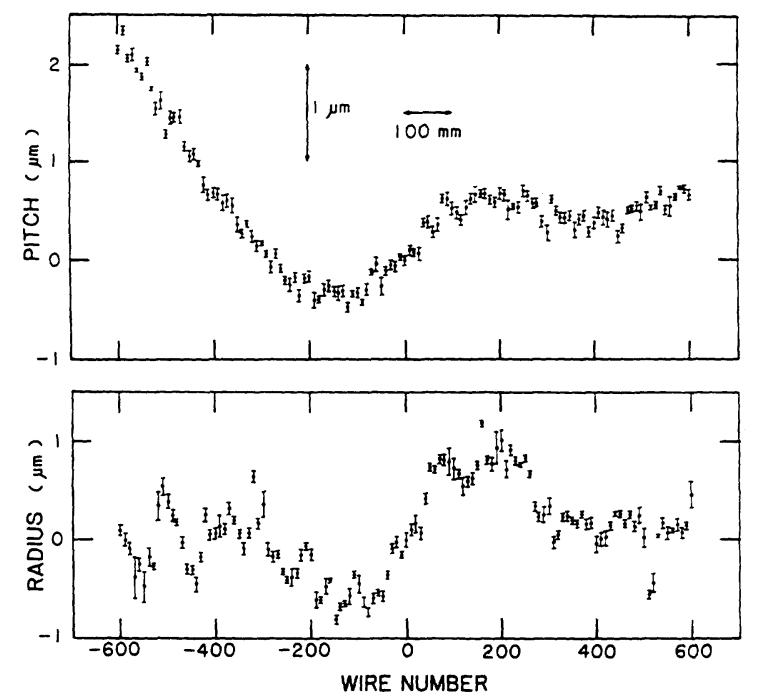

Fig. 4. Radial variations (bottom) in $\mu \mathrm{m}$ and axial position variations (top) in micrometers versus wire number $(\sim 1 \mathrm{~mm} /$ wire number). The radial variations are with respect to the center wires. For the axial variations a uniform pitch of $1.058588936 \mathrm{~mm} /$ turn has been subtracted from the laser interferometer reading at each wire number. The error bars are meant to represent one standard deviation uncertainty estimates.

to the measurement uncertainty of the outer windings is significantly less. Current is injected into 10 turns at a time, so that the data plotted is the average position of the ten turns. The axial variations are a measure of turn position after a uniform pitch of $1.058588936 \mathrm{~mm} /$ turn has been subtracted from the laser readings of that position. The radial variations are a plot of the difference in radius of the ten turns at the indicated axial position relative to the center ten turns. These measurements show that the current in the solenoid flows within a few micrometers of where current in a perfect solenoid would flow.

\section{The Diameter Problem}

With the high accuracy of the radius variation and pitch measurements, the diameter measurement should be the limiting factor in the experiment. The basic problem in determining the diameter is that even if one makes a perfect measurement of the position of the surface of the copper wire, it is still difficult to know where the current flows in the wire. The technique developed to eliminate the need for a diameter measurement is to find a coil geometry which produces a magnetic field that is nearly independent of the average diameter. With a long solenoid we are able to find a suitable configuration in which extra current can be added to selected turns near the end of the solenoid. If done properly, this, in effect, gives us the same properties of an infinitely long solenoid; namely, that the magnetic field is independent of diameter and is uniform at the center. With the aid of a computer, we found a configuration where the sum of the radius-variation weighting factor for each turn approaches zero, and the second-, fourth-, and sixth-order gradients are compensated. The result (see Fig. 5) is that our solenoid can produce a field that has the same diameter dependence as a $1-\mathrm{km}$ long solenoid (i.e., the uncertainty due to the diameter measurement is essentially

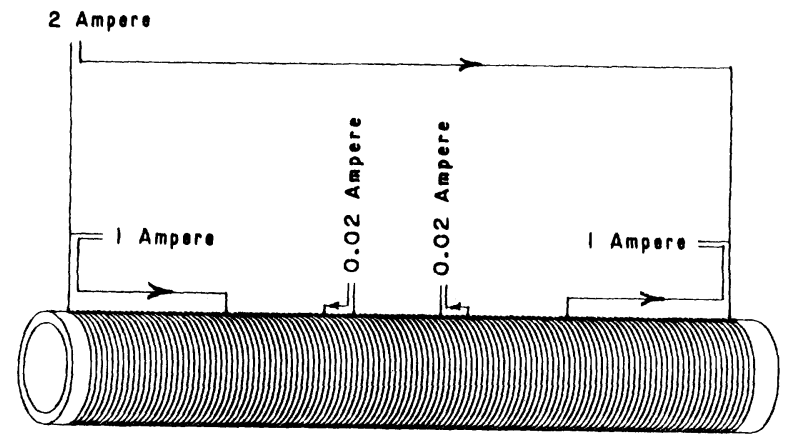

Fig. 5.: Compensation scheme for obtaining an "infinite" solenoid for NMR measurements. By using five current sources connected as indicated, a magnetic field is produced that is both uniform to 2 parts in $10^{8}$ over a $6-\mathrm{cm}$ sphere and essentially independent of the average diameter of the solenoid. Leakage between the five current sources must be kept very low.

eliminated) and a field that is uniform to 2 parts in $10^{8}$ over a $6-\mathrm{cm}$ diam spherical volume at the center of the solenoid. (The water sample for the NMR measurements need only be $4 \mathrm{~cm}$ in diameter.)

\section{OTHER IMPROVEMENTS}

There is a remote chance that leakage between two adjacent turns will cause an error in the calculated field. For our single helix solenoid a resistance of $30 \mathrm{k} \Omega$ across the most critical wires will result in a $0.01 \mathrm{ppm}$ error. The problem is that the low resistance of the winding makes such leakage difficult to detect. The technique we have developed to avoid this problem is very simple: We inject a dc current $(5 \mathrm{~A})$ into each turn, one turn at a time. If there is leakage to an adjacent turn, a leakage current will flow in it. However, the finite resistance of that turn produces a small voltage drop that can be detected with a pair of potential leads connected to the neighboring wire. A leakage of $30 \mathrm{k} \Omega$ gives a voltage of $3 \times 10^{-8} \mathrm{~V}$; and using a sensitive null detector and an automated system, we can test every wire.

The other major problem that we plan to solve is the correction for the magnetic susceptibility of the fused silica cylinder. We have taken three core samples and from their measured small susceptibilities have calculated that the correction to the magnetic field is very small $(<0.01 \mathrm{ppm})$, but this assumes that the form is homogeneous. To verify that it is in fact homogeneous, we must measure the susceptibility correction directly. To this end, we have constructed a large $(0.6 \mathrm{~m}$ diam by $2.2 \mathrm{~m}$ long) solenoid wound on a fused-silica cylinder. We will measure changes in the field produced by this large solenoid as we lower our precision solenoid in and out of this field. We must detect changes of $0.01 \mathrm{ppm}$ to measure the susceptibility correction to the same accuracy.

\section{CONCLUSION}

We are presently measuring our new solenoid with an accuracy of $0.05 \mathrm{ppm}$. We have a precision of $0.02 \mathrm{ppm}$ and expect to improve the accuracy of the measurements to this level.

We plan to carry out the NMR part of the experiment within the next four to six months. This should give us a $0.1 \mathrm{ppm}$ preliminary value of $\gamma_{p}^{\prime}$. We then plan to spend another year 
testing for systematic effects before obtaining final results. Ultimately we hope to obtain a value of $\gamma_{p}^{\prime}(\text { low })_{\text {NBS }}$ with an uncertainty of $0.05 \mathrm{ppm}$ or less, which should yield a value of $\alpha$ with an uncertainty of about $0.02 \mathrm{ppm}$.

\section{ACKNOWLEDGMENT}

The authors would like to thank E. Muth and D. Trammell for their dedication in helping to construct the solenoid; $\mathrm{H}$. Layer who calibrated the laser; G. Barrett who helped analyze data; K. Maruyama, an NBS Guest Scientist from the Tokyo Institute of Technology, Japan, who designed the injector probe, and F. Fickett, of NBS/Boulder, for measuring the susceptibility of the solenoid form material.

\section{REFERENCES}

[1] E. R. Williams and P. T. Olsen, "New measurement of the proton gyromagnetic ratio and a derived value of the fine-structure constant accurate to a part in $10^{7}, "$ Phys. Rev. Lett., vol. 42 , pp. 1575-1579, June 1979.

[2] E. R. Williams, P. T. Olsen, and W. D. Phillips, "The proton gyromagnetic ratio in $\mathrm{H}_{2} \mathrm{O}$-a problem in dimensional metrology," in Precision Measurement and Fundamental Constants II, Natl. Bur. Stand. (U.S.), Spec. Publ. 617 (USGPO, Washington, DC), B. N. Taylor and W. D. Phillips, Eds., 1984, pp. 497-504.

[3] H. L. Curtis, C. Moon, and C. M. Sparks, "A determination of the absolute Ohm, using an improved self inductor," J. Res. Natl. Bur. Stand., vol. 21, pp. 375-423, Oct. 1938, and private communication with R. L. Driscoll.

[4] E. R. Williams and P. T. Olsen, "A noncontacting magnetic pickup probe for measuring the pitch of a precision solenoid," IEEE Trans. Instrum. Meas., vol. IM-21, pp. 376-379, Nov. 1972.

[5] H. P. Layer, "A portable iodine stabilized helium-neon laser," IEEE Trans. Instrum. Meas., vol. IM-29, pp. 358-361, Dec. 1980.

\title{
First Results of the $\gamma_{p}^{\prime}$ Experiment at the PTB
}

\author{
KURT WEYAND
}

\begin{abstract}
A $\gamma_{p \text {,low }}$ experiment based on a novel method of determining the coil constant has yielded preliminary results. The coil constant is found by measuring the flux profile along the axis of the windings. When the NMR experiment is performed in the free precession mode, the value of $\gamma_{p}$ amounts to $\gamma_{p}^{\prime}=2.675143 \times 10^{8}\left( \pm 3.4 \times 10^{-6}\right)$ $\mathrm{T}_{\mathrm{PTB}}^{-1} \cdot \mathrm{s}^{-1}$ with a $(1 \sigma)$ uncertainty.
\end{abstract}

\section{INTRODUCTION}

$\mathrm{T}$ HE MOST important problem in carrying out a $\gamma_{p}$ experiment in low mangetic fields is to determine the value of the flux density acting on the NMR probe. Up to now the dimensions of single-layer coils have been measured by mechanical and optical methods [1], [2], sometimes in combination with electromagnetic indicator systems [3], and from the results of those measurements the flux density at the center of the field coil has been evaluated. Whatever procedure is used, in order to determine the diameter of these coils a comparison with an end gauge must be performed, and this may be an important source of error. Added to this, even if the positions of the layer are well known, there is another source of error due to the current distribution in the wire, which is influenced by the wire's history and chiefly by the winding procedure.

For this reason a novel method based on measuring the effect of current flowing in multilayer coils has been developed in order to find the coil constant of a system of coils, i.e., as it

Manuscript received August 20, 1984.

The author is with the Physikalisch-Technische Bundesanstalt, Bundesallee 100, D-3300 Braunschweig, Federal Republic of Germany.

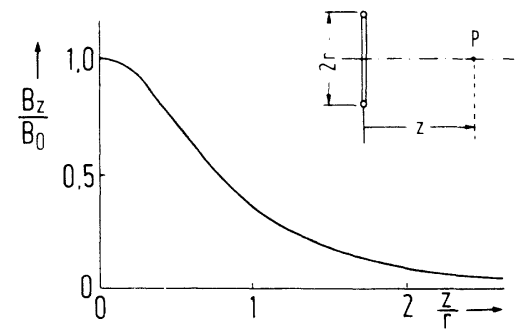

Fig. 1. Method for determining the effective radius of a current loop by relative field measurement.

is understood here, the value of flux density at the center of the system as a function of the current through the coils.

\section{Determination of the Coll Constant}

The principle of this method can be made clear by a simple example. Assuming a single circular turn with radius ' $r$ ' as shown in Fig. 1 the flux density $B_{z}$ along the $z$-axis is given by

$$
B_{z}=\frac{\mu I}{2 r}\left[1+(z / r)^{2}\right]^{-3 / 2} \text {. }
$$

Thus the only unknown geometric dimension ' $r$ ' may be evaluated by measuring the field $B_{0}$ at the center and $B_{z}$ at any other point $P$ on the axis of the turn at a distance $z$ and by forming the field ratio $V_{z}=B_{0} / B_{z}$, giving:

$$
r_{\mathrm{eff}}=z\left[V_{z}^{2 / 3}-1\right]^{-1 / 2}
$$

where all $B$ values may be measured in arbitrary units. Thus 